\title{
Researching the Behavior of Pedestrian Based on Social Force Model in Urban Rail Channel
}

\author{
Tianyang $\mathrm{Qi}^{1, a}$, Xiangyong Yin ${ }^{2, \mathrm{~b}}$ \\ ${ }^{1}$ School of Traffic and Transportation, Beijing Jiaotong University, Beijing 100044, China \\ ${ }^{2}$ School of Traffic and Transportation, Beijing Jiaotong University, Beijing 100044, China \\ a15120767@bjtu.edu.cn, bxyyin@bjtu.edu.cn
}

Keywords: social force model; urban rail transit; pedestrian flow

Abstract. In order to simulate the pedestrian flows in the normal conditions realistically, on the basis of the existing social force model, the phenomenon in the original simulation such as collision avoidance between pedestrian trajectory is too large, the pedestrian path is not smooth, the collision of the pedestrian is overlap, and collision is too large between pedestrians and wall are improved, making the simulation program more realistic. Through the simulation experiment, the effectiveness of the improvement of social force simulation model is verified.

\section{Introduction}

Pedestrian traffic is an important part in urban traffic. In urban rail transit stations, there is also a large scale of pedestrian act. Much streamline intersections and conflicts will especially be produced when pedestrians are walking in channels, which can influence their walking time as well as the traffic capacity and service level of cross regions. Study on the pedestrian walking characteristics in urban rail transits can effectively improve the walking efficiency and safety coefficient of them in the rail transit. Therefore, the research on the pedestrian walking act is gradually becoming a key topic concerned by society currently.

Study on the pedestrian simulation model by scholars has achieved certain results. The pedestrian simulation model can be divided into the macroscopic model and microscopic model. The macroscopic model treats pedestrians as continuously flowing media and carries out the analogue simulation by making use of the fluid mechanics. However, the interaction between the individual difference and individuals is neglected. The pedestrian traffic flow model was initially proposed by L.F. Henderso[1]. He used the fluid dynamics to simulate the pedestrian traffic flow, thus analyzing the influence of sex, age, starting point and finishing point of travel and other factors on speed distribution. The pedestrian speed probability distribution formula was also proposed. The fluid dynamics model is rather representative among macroscopic pedestrian flow simulation. The social force model put forward by Helbing is with significant advantages, which attracts numerous scholars to study and improve it. In addition to the verification and amendment of basic model parameters, the pedestrian prediction mechanism, attraction interference, barrier bypass, initiative avoiding, companion groups and other factors are also involved in, in order to enhance the traditional social force model.

Through the amendment of view angles and stride sizes Luo Qian[5] introduced the contact force to eliminate overlap. Ding Qingyan[6] et al. improved the driving force, attraction and direction influence in model parameters. By taking the automatic canalization phenomenon and the arch formed in the exit of rail transit pedestrian flow movement as an example, the effectiveness of the model is illustrated. To resolve the pedestrian overlap problem, Li Shanshan[7] introduced the pedestrian deceleration avoiding mechanism. When the pedestrian density is certain, the pedestrian flow movement is simulated. On the basis of the gravity model, $\mathrm{Fu} \mathrm{Yu}$ [8] added the social force model thought and established the rail transit pedestrian flow gravity simulation model. When pedestrians meet obstacles, they can avoid obstacles more smoothly, thus making the pedestrian movement track more realistic. Wang Zijia[9] introduced the molecular dynamics algorithm in the social force model to simulate the unidirectional and bidirectional pedestrian movement in the rail 
transit station channels, so as to reach the automatic pedestrian stream canalization effect. By introducing the pedestrian avoiding collision mechanism, Zhang Haijun and Shi Huaji[10] adopted two different collision avoidance mechanisms for pedestrians in the same direction and in different directions, thereby improving the social force model.

Based on the pedestrian traffic flow theories and social force model study by scholars, this paper attempts to improve weaknesses of the existing simulation models according to pedestrian walking characteristics in the pedestrian channels of urban rail transit stations. Additionally, the simulation model is applied to the subway channel simulation of different contexts.

\section{Problems of the initial model}

The initial social force model. The social force model proposed by Helbing is one of the pedestrian microscopic simulation mechanical models. Through the social force effect, some displacement is conducted by pedestrians. Pedestrians receive three forces in the social force model: the self-driving force produced by individuals attracted by the destination $\overrightarrow{F_{\mathrm{WD}}}$, the interaction force with other pedestrians $\overrightarrow{F_{1 p}}$, the acting force from the external environment $\overrightarrow{F_{1 W}}$ (e.g. the force between pedestrians and obstacles). The combined three forces $\vec{F}$ is the final force received by pedestrians:

$$
\vec{F}=\overrightarrow{F_{\mathrm{ID}}}+\overrightarrow{F_{\mathrm{ip}}}+\overrightarrow{F_{\mathrm{IW}}}
$$

The acceleration of pedestrians at this moment can be calculated by the combined force, and the next moment speed of pedestrians can be calculated as well:

$$
\begin{aligned}
& a=F / m_{\mathfrak{i}} \\
& v_{\mathrm{i}}(t)=a t+v_{0}(t)
\end{aligned}
$$

Where ${ }^{a}$ is the acceleration of pedestrians; $m_{i}$ is the quality of pedestrian $i$; $v_{i}(t)$ is the speed of pedestrians at the moment $t$; $v_{0}(t)$ is the initial speed of pedestrians; ${ }^{t}$ is the time.

Existing problems. Lakoba believes that the expected speed direction of pedestrians is not only related to the target point, but also affected by the speed of surrounding pedestrians. This influence is with a certain range which is described as a circle taking the pedestrian as the center, thus calculating the mean speed of pedestrians within the circle range. In the traditional social force model, it is considered that the influence of pedestrian surrounding is the same on them, while the anisotropy of them is neglected. In fact, when there are other pedestrians showing around the pedestrian, he or she will change his or her own target route in accordance with other pedestrians' walking direction, and the route is rather smooth. It is relevant with the vision scope and expected speed direction of pedestrians.

Although pedestrian movement simulation can be realized via three acting forces by the traditional social force model, there are still some weaknesses: the expected speed is not a certain value. Due to the limitation of the study object, the specific walking relationship between two pedestrians is neglected, so does the factor whether both sides can see each other. When two pedestrians walk in opposite directions, they cannot see each other, so there is none psychological exclusion force interaction existing between the two persons. However, in the traditional social force model, it is considered that there is interaction between two persons within a certain range. When two persons contact each other in opposite directions, it will produce excessive overlap. Furthermore, due to the suddenly produced repellent force and friction force, the two persons will be bounced off, which will lead to the significant retrogression act of them. In the traditional social force model, influenced by different degrees of acting forces in opposite directions on both sides of the wall, irrational shaking is produced in channels when pedestrians are walking there.

\section{The Improvement of Social Force Simulation Model}

Improved force between pedestrians and obstacles. In the traditional social force model, the great shear force between the pedestrian and the wall causing pedestrians will be bounced off after impact the wall. In order to make the repulsive force in the simulation model of collision between the 
obstacle and pedestrian more reasonable, to improve the force between the pedestrians and obstacles in formula1. The improvement mainly reflected in the two recalibrated coefficients $A_{w}, B_{w}$ which in the external environment forces for calibration procedures. Improved parameters by experiment, and changed distance boundary produced by obstacle friction $0.3 \mathrm{~m}$ from $0.2 \mathrm{~m}$. The changed formula is as follows:

$$
\overrightarrow{F_{\text {IW }}}=\overrightarrow{F_{n w}^{F}}+\overrightarrow{F_{w w}^{p}}
$$

Pedestrians always maintain a certain distance with obstacle when they are walking. Pedestrians will have a psychological repulsive force to obstacle when distance between obstacles close but not touching.

$$
\overrightarrow{F_{\mathrm{nV}}}=A_{\mathrm{W}} \exp \left(\frac{\vec{y}_{\mathrm{i}}-d_{\mathrm{in}}}{\vec{B}_{\mathrm{W}}}\right) \overrightarrow{n_{\mathrm{nW}}}
$$

Where $A_{w}$ is the interaction force strength between pedestrians and obstacles; $B_{w}$ is the interaction range between pedestrians and obstacles; $d_{\text {iw }}$ is the distance between pedestrians and obstacles; $\overrightarrow{n_{\mathrm{nw}}}$ is a unit normal vector directed from obstacle to pedestrian.

When pedestrians and obstacles cannot avoid contact, pedestrians will also be given a physical force of obstacles makes pedestrians away from obstacles as soon as possible. At this time, the physical forces are the pedestrian's positive pressure $\overrightarrow{f_{w}^{p}}$ from obstacle and sliding friction $\overrightarrow{f_{n w}^{f}}$.

$$
\overrightarrow{F_{i w}^{p}}=K \Theta\left(r_{i}-d_{i w}\right) \overrightarrow{n_{i w}}+k \Theta\left(r_{i}-d_{i w}\right)\left|v_{i}\right| \cos \left\langle v_{i}, \overrightarrow{t_{i w}}\right\rangle \overrightarrow{t_{i w}}
$$

Where $\overrightarrow{t_{\mathrm{nv}}}$ is a unit tangent vector pointing pedestrians' direction; $v_{\mathrm{i}}$ is the speed of pedestrians; $\cos \left\langle v_{i}, \overrightarrow{t_{\mathrm{n}}}\right\rangle$ is the angle between pedestrian speed and unit tangent vector. The post-correction parameters, see Table 1.

Table 1. simulation parameters correction

\begin{tabular}{|c|c|c|c|}
\hline Name & Symbol & Size & Unit \\
\hline $\begin{array}{c}\text { Force Strength Between Pedestrians And } \\
\text { Obstacles }\end{array}$ & $A_{w}$ & 150 & $\mathrm{~N} / \mathrm{m}$ \\
\hline Force Range Between Pedestrians And Obstacles & $B_{w}$ & 0.5 & $\mathrm{~m}$ \\
\hline Distance Limit Of Friction To Obstacles & $d_{m}$ & 0.3 & $\mathrm{~m}$ \\
\hline
\end{tabular}

Improvement of pedestrians' overlapping. Pedestrians' overlapping may happen in the traditional model because of unavoidable collision. Of which, some happen in channels, some near the entrances or exits of channels and some between pedestrians walking in the opposite or the same direction. Two suggestions were provided to improve this phenomenon.

(A) Introduction of the pedestrian overlapping mechanism

There existed no effective method to solve this question in the traditional social force model, which includes overlapping between two pedestrians or between pedestrians and obstacles. Therefore, to prevent above-mentioned abnormal overlapping, this paper hereby introduced the pedestrian overlapping mechanism. In the Lakoba simulation model, pedestrians were described as a circle with a radius of $r$, while the maximal outer extrusion amount that exerted on the pedestrians was described as $S_{\max }$, which was $20 \%$ of the r. When the amount was less than or equal to this value, the overlapping was normal, but once the amount was more than the value, overlapping appeared. To judge this phenomenon, the moving directions of the pedestrians and the number should be taken into consideration.

(1) if two pedestrians (marked as $i$ and $j$ respectively) walked in the same direction, if $j$ was in the front, then $\mathrm{i}$ stopped moving and $\mathrm{j}$ kept moving, when their distance was more than the sum of their radius, $\mathrm{i}$ began to move.

(2) if two pedestrians (marked as $\mathrm{i}$ and $\mathrm{j}$ respectively) walked in the opposite directions according to their number, the smaller one kept moving, the bigger one stopped first till their distance was more than the sum of their radius.

This theory was also applied to the overlapping between pedestrians and obstacles. When this overlapping appeared, dividing the speed of the pedestrians into normal velocity and tangent velocity according to the directions of the obstacles. Decreasing the normal velocity to 0 can prevent 
pedestrians from moving close to obstacles, in the meantime, the tangent velocity remained unchanged. In the next step calculation, the force from the obstacles would generate the normal velocity away from obstacles, and pedestrians would move away from the obstacles gradually.

(B) Addition of one constraint condition in the entrances and exits of channels

In the entrances and exits of the channel, pedestrians who just entered the channel would collide with those who were leaving, thus, one constraint condition should be added here. When $i$ entered the constraint area and was getting out of the channel (the left side of the constraint area was taken as $\mathrm{x}$-axis $[0,0.5]$ and the right side $[9.5,10]$ ), reading the $\mathrm{y}$ axis coordinate information of $\mathrm{j}$. If the absolute value of the difference between their ordinates was less than the sum, using the ordinate of $j$ to add or subtract the sum of their radius, following was the detailed process:

The first step: $\left|y_{i}-y_{j}\right| \leq r_{i}+r_{j}$, then $y_{j}^{\mathrm{n}}=y_{j}-2 r_{j}$;

The second step: if the achieved $y_{j}^{n} \leq 0$, then $y_{j}^{n}=y_{j}-4 r_{j}$. $\left(y_{j}^{n}\right.$ is the newly generated coordinate in $y$ axis of $j$ )

Improvement on pedestrian avoidance and repulsion. This part improved the unreasonable phenomena including collision caused by pedestrian avoidance or early pedestrian avoidance in the simulation. In order to drive the pedestrian's walking path to better conform to the reality, an avoidance angle was designed to improve the model.

A deflection velocity was offered to one of the pedestrians before the collision aiming to move laterally to avoid another opposite direction pedestrian. As shown in figure 1, A will collide with B face to face. When the distance between $\mathrm{A}$ and $\mathrm{B}$ conforms to the action range of deflection angle, $\mathrm{A}$ will have a longitudinal velocity to avoid the imminent collision. A rectangular frame was set up for the avoidance scope with the horizontal one of $0.8 \mathrm{~m}$ and vertical one of $0.5 \mathrm{~m}$. The specific calculation for deflection coefficient angle, see formula 7.

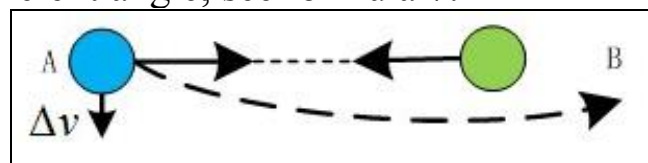

$\theta=\mathrm{x}-\left|y_{j}-y_{i}\right| /\left|x_{j}-x_{i}\right|$

Fig 1. Pedestrians bypassing trajectory

Multiplied $\theta$ and the pedestrian speed on $\mathrm{y}$ axis and the deflection velocity of the $\mathrm{y}$ axis is obtained.

Formation of Pedestrian's Self-Organization Phenomenon after Improvement. It was found through the research on pedestrian's walking behavior on urban railway transit hub that the pedestrians will follow the man in front on the two-way pedestrian walkway by forming several main pedestrian paths when with large pedestrian flow. The walking directions on every path are similar with the pedestrian flow automatic channelization to be an ordered state from the previous chaotic state. This phenomenon improved pedestrian's walking efficiency and avoid conflict and collision among pedestrians at opposite direction effectively.

\section{Simulation}

This paper built the simulation model concerning the pedestrian's social force model by using MATLAB software as the tool, conducted the simulation in inner channel by taking the pedestrian channels in urban railway transit hub as the background and then applied the simulation in the real rail transit station channel.

Parameter Calibration.Every pedestrian was described as circles with different radius in MATLAB simulation system. This paper calibrated the pedestrian's quality, radius, initial expected velocity, initial actual velocity, pedestrian's relaxation time and field of vision according to the analysis on the characteristics of pedestrian flow in chapter 2 as well as the investigation on the pedestrian's basic parameters in relevant information. Please see Table 2.

Of which, the pedestrian's quality range was $[50 \mathrm{~kg}, 80 \mathrm{~kg}]$, which obeyed the normal distribution $\mathrm{N}\left(65,5^{2}\right)$; the pedestrian's radius range was $[0.18 \mathrm{~m}, 0.28 \mathrm{~m}]$, which obeyed the normal distribution 
$\mathrm{N}\left(0.23,0.5^{2}\right)$; further, the initial expected velocity and initial actual velocity obeyed the uniform distribution.

As shown in the data, the pedestrian's relaxation time was often between $0.1 \mathrm{~s}$ and $0.5 \mathrm{~s}$, which would be $1-3 \mathrm{~s}$ under complex condition. Here was taken $0.5 \mathrm{~s}$ as the fixed relaxation time of pedestrians.

Table 2. pedestrian's basic parameters

\begin{tabular}{|l|c|c|c|}
\hline Name & Symbol & Size & Unit \\
\hline quality & $m$ & $50-80$ & $\mathrm{~kg}$ \\
\hline radius & $r$ & $0.18-0.28$ & $\mathrm{~m}$ \\
\hline initial expected velocity & $v_{0}$ & $1.35-1.45$ & $\mathrm{~m} / \mathrm{s}$ \\
\hline initial actual velocity & $v$ & $1.2-1.6$ & $\mathrm{~m} / \mathrm{s}$ \\
\hline field of vision angle & $\theta$ & 180 & $\circ$ \\
\hline field of vision radius & $R$ & 6 & $\mathrm{~m}$ \\
\hline
\end{tabular}

According to the simulation environment, relevant information and rationality analysis, the simulation system's relevant parameters setting, see Table 3.

Table 3. simulation system's parameters

\begin{tabular}{|l|c|c|c|}
\hline Name & Symbol & Size & Unit \\
\hline Channel Width & $a$ & 3.6 & $\mathrm{~m}$ \\
\hline Channel Length & $b$ & 10 & $\mathrm{~m}$ \\
\hline Force Strength Between Pedestrians & $A_{\eta}$ & 200 & $\mathrm{~N} / \mathrm{m}$ \\
\hline Force Range Between Pedestrians & $B_{\eta}$ & 8 & $\mathrm{~m}$ \\
\hline Force Strength Between Pedestrian and Obstacle & $A_{\mu}$ & 200 & $\mathrm{~N} / \mathrm{m}$ \\
\hline Force Range Between Pedestrian and Obstacle & $B_{\mu}$ & 0.2 & $\mathrm{~m}$ \\
\hline Positive Pressure Coefficient & $k$ & 12000 & $\mathrm{~kg} / \mathrm{s}^{2}$ \\
\hline Sliding Friction Coefficient & $K$ & 24000 & $\mathrm{~kg} / \mathrm{s}^{2}$ \\
\hline Relaxation Time & $\tau$ & 0.5 & $\mathrm{~s}$ \\
\hline Pedestrian's Weight Coefficient & $\lambda$ & 0.2 & - \\
\hline Conformity Weight Coefficient & $\rho$ & 0.3 & - \\
\hline Simulation Step & $t$ & 0.1 & $\mathrm{~s}$ \\
\hline Predicted Range Width Between Pedestrians & $a_{\alpha}$ & 1 & $\mathrm{~m}$ \\
\hline Predicted Range Length Between Pedestrians & $b_{\alpha}$ & 3 & $\mathrm{~m}$ \\
\hline
\end{tabular}

Helbing[1],[3] introduced positive pressure coefficient $k$ and sliding friction coefficient $\mathrm{K}$ in the traditional social force model. Those two coefficients were increased to avoid the overlapping at the time of collision among pedestrians. However, the excessive coefficients will cause the calculated pressure value not to conform to the reality. Here those two coefficients were decreased by taking the sums respectively.

The pedestrian's weight coefficient $\lambda(0.2)$ was introduced at the time of calculating the psychological repulsive force aiming to reduce the force of the pedestrians behind on the ones in front properly. The conformity coefficient $\rho$ in the expected velocity was equal to 0.3 .

The avoidance mechanism among pedestrians could be achieved according to the pedestrian predication mechanism in the social model put forward by Loannis Karamouzas. The prediction range meant the pedestrians within $3 \mathrm{~m}$ from normal vector and $1 \mathrm{~m}$ from tangent vector of the predicted velocity. That is to say, a rectangle frame (width: $2 \mathrm{~m}$, length: $6 \mathrm{~m}$ ) that takes the pedestrians as the center; the intensity and range of applied force among pedestrians were recalibrated according to the pedestrian predication mechanism. The predication mechanism among pedestrians was introduced into the interaction between pedestrian and obstacles. Further, the intensity and range of applied force between pedestrian and obstacle were recalibrated. 


\section{Result analysis.}

(A) Interaction between pedestrians and obstacles

The walking trajectory of No4 pedestrian in simulation after correcting the parameters, see Figure 2.

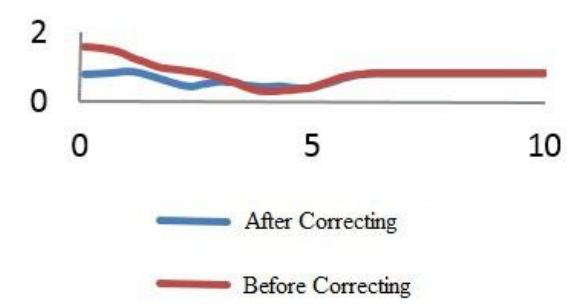

Fig 2. Trajectory of No.4 pedestrian

The simulated phenomenon after correcting the parameters showed that No 4 pedestrian got close to the wall at about 5 on $\mathrm{x}$ axis, then walked away from the wall slightly due to its repulsive force, later got close to the wall in order to avoid other pedestrians and then walked away from the wall again. Finally, No.4 pedestrian came out of the channel at about 0.8 on y axis. On the contrary, the simulation phenomenon before the improvement showed that No 4 pedestrian kept far away from the wall after bouncing off and then came out of the channel at about 1.6 on y axis. It can be observed from the above two mentioned simulated effects that the corrected coefficient can bring out more reasonable force between the pedestrian and obstacle.

(B) Simulation on pedestrian overlapping phenomenon

Aiming at the improvement of pedestrian overlapping phenomenon, the MATLAB programming was conducted to obtain new simulation on pedestrian walking in the experiment channel in Xizhimen metro. No.26 pedestrians and No.35 pedestrians were supposed to have collision and overlapping. However, in fact, No.26 pedestrian came out from the left channel, while No 35 pedestrians just came in the channel as shown in figure 3 without any collision and overlapping due to the previously mentioned access limit and overlapping mechanism, which indicated that the above mentioned two improvements have better effect on the pedestrian collision and overlapping phenomena. It corresponds to the practical situation basically. The avoidance trajectory of No 26 and 35 pedestrians, see Figure 4.

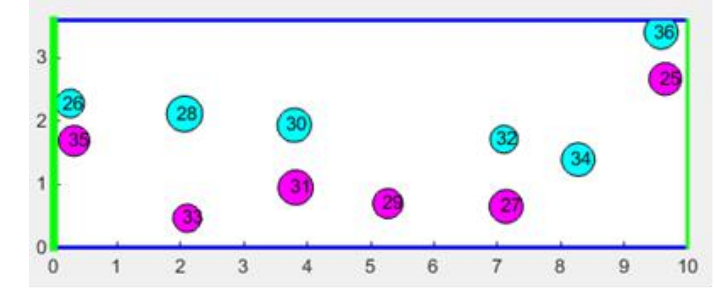

Fig 3. Pedestrian avoidance phenomenon

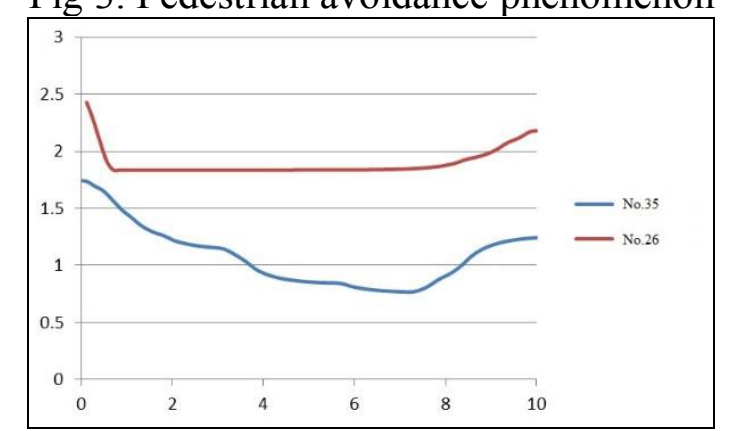

Fig 4. The trajectory of No.26 and No.35 pedestrians

(C) Simulation on Pedestrian's Avoidance and Repulsion

The simulation on pedestrian's avoidance and repulsion was conducted in accordance with the improved suggestions to obtain the walking paths of top 10 pedestrians in Figure 5, whose walking trajectory were basically smooth with better avoidance effect. Further, it can be observed from the pedestrian's velocity data that the unreasonable phenomena including sharp acceleration and sharp deceleration etc were not occurred in the velocity-change process. 


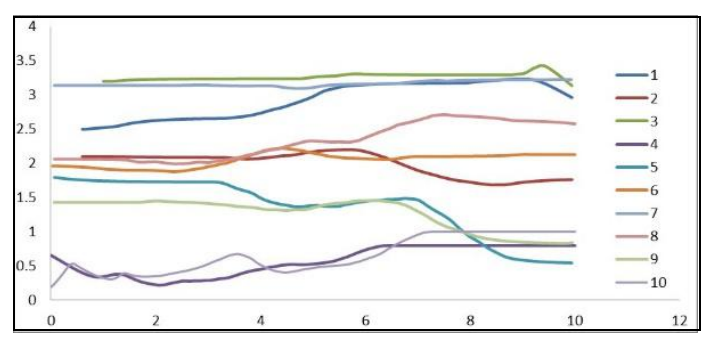

Fig 5. Walking paths of top 10 pedestrians

\section{Conclusion}

As an important component of urban traffic, the pedestrian traffic has been the research concern of various scholars. With the rapid development of urban rail transit, the researches on the characteristics and rules of pedestrian flow in the rail transit stations can provide theoretical foundation for the stream of people, service level and safety measures.

This paper established the pedestrian simulation system of inner channel of urban rail transit based on MATLAB software as well as scholar's research and improvement on social force model by analyzing and studying the characteristics of pedestrian flow and inner channels in urban rail transit and then improved the phenomena including too large avoidance trajectory, unsmooth walking trajectory, overlapping after collision and bouncing off after the collision with walls etc through reasonable parameter setting, simulation experiments and analysis aiming to bring out more practical social force simulation system.

\section{References}

[1]Hongfei Jia, Lili Yang, Ming Tang. Pedestrian Flow Characteristics Analysis and Model Parameter Calibration in Comprehensive Transport Terminal[J]. Journal of Transportation Systems Engineering and Information Technology,2009,9(5):117-123.(in Chinese)

[2]Helbing D A mathematical model for the behavior of pedestrians[J]. Behavioral Science, 1991,36(4):298-310.

[3]Helbing D . A fluid-dynamic model for the movement of pedestrians[J] . Complex Systems, 1992,6(5):391-415.

[4]Helbing D,Molnar P. Social force model for pedestrian dynamics[J] . Physical Review E,1 995,51(5):4282-4285.

[5]Qian Luo. Pedestrian Evacuation Model Correction and Simulation Research in Social Force [D]. Capital University of Economics and Business,2010. (in Chinese)

[6]Qingyan Ding, Xifu Wang, Qingchao Shan, Xiuyuan Zhang. Modeling and Simulation of Rail Transit Pedestrian Flow[J]. Journal of Transportation Systems Engineering and Information Technology, 2011,11(5). (in Chinese)

[7]Shanshan Li, Dalin Qian, Jiuzhou Wang. Improved social force model considering pedestrian deceleration to avoid collision [J]. Journal of Jilin University Engineering and Technology Edition, 2012,42(3):623-628. (in Chinese)

[8]Yu Fu. Modeling and Simulation of Rail Transit Station Pedestrian Flow [D].Beijing Jiaotong University,2012. (in Chinese)

[9]Zijia Wang, Feng Chen, Zhongheng Shi. Agent-Based Realization of Social Force Model and Simulation of Pedestrians in Subway Passageway[J].Journal of South China University of Technology(Natural Science Edition),2013,41(4):90-94. (in Chinese) 
[10]Haijun Zhang, Huaji Shi.Research of bi-directional pedestrian flow's model on modification of social force [J]. Computer Engineering and Applications, 2013(16) :236-239. (in Chinese)

[11]Lakoba T I,Kaup D J,Finkelstein N M.Modifications of the Helbing-Molnar-Farkas-Vicsek socialforce model for pedestrian evolution[J]. Simulation-transactions of the Societyfor Modelingand Simulation International,2005,81(05):339-352.

[12]Qingchao Shan. Modeling and Simulation of Pedestrian Flow Movement in Urban Rail Transit[D]. Beijing Jiaotong University,2009. (in Chinese)

[13] Shouhua Cao. Analysis and Modeling Urban Rail Transit Passengers Traffic Characteristic [D]. Beijing Jiaotong University,2009. (in Chinese)

[14]Xiaochuan Tian. Application of the improved social force model in comprehensive transportation facilities efficiency analysis [D]. Jilin University,2012. (in Chinese)

[15]Karamouzas, I,Heil, P,van B P. A Predictive Collision Avoidance Model for Pedestrian Simulation.[J].Zeist :2nd International Workshop on Motion in Games,2009(5884):41-52. 